\title{
Psychological function is mildly impaired for prolonged periods before and after first unipolar major depressive episode
}

Ormel J, Oldehinkel AJ, Nolen WA, et al. Psychosocial disability before, during, and after a major depressive episode. A 3-wave population-based study of state, scar, and trait effects. Arch Gen Psychiatry 2004;61:387-92.

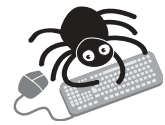

\section{Is psychosocial disability present before and after a person's first unipolar major depressive episode?}

\section{METHODS}

$\square$

Design: Prospective longitudinal study

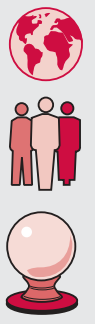

Setting: General population, the Netherlands; enrolment 1996-99.

20 Population: 4796 people aged 18-64 years in 1996, randomly sampled from the general population. People with bipolar disorders were excluded.

Prognostic factors: Psychiatric data were obtained by Composite International Diagnostic Interview, as part of the Netherlands Mental Health Survey and Incidence Study (NEMSIS).

Psychosocial function was assessed using the employment, partner, housekeeping, and leisure scales of the Groningen Social Disability Schedule. Between subject and within subject differences in disability were compared using Student's $t$ test. Retest and time effects were tested using ANOVA. Differences were expressed as effect sizes, which were calculated by dividing the mean difference in disability by the standard deviation of disability in the two groups.

Follow up period: One and three years.

\section{MAIN RESULTS}

At three years, 216 people had developed and recovered from a first major depressive episode, while 118 people had developed and recovered from a recurrent major depressive episode (MDE) (see http://www.ebmentalhealth.com/supplemental for table 1). People recovering from a first MDE had higher disability scores long before their episode than people who never had an MDE (see table 2). Disability scores among people who had an MDE were similar before and after the episode, except for people suffering severe recurrent

Table 2 Comparison of disability scores for people with remitted first major depressive episode (MDE) and no MDE at three years

\begin{tabular}{lll}
\hline Psychosocial disability scales & Effect size & $95 \% \mathrm{Cl}$ \\
\hline Leisure & 0.42 & 0.27 to 0.56 \\
Employment & 0.44 & 0.26 to 0.62 \\
Housekeeping & 0.50 & 0.34 to 0.66 \\
Partner & 0.57 & 0.39 to 0.75 \\
Total score & 0.50 & 0.35 to 0.65 \\
\hline
\end{tabular}

For correspondence: Johan Ormel, Department of Psychiatry, University of Groningen, Groningen, the Netherlands; i.ormel@med.rug.nl

Sources of funding: the Netherlands Ministry of Health, Welfare and Sport, The Hague; The Netherlands Organization for Scientific Research, The Hague; and the National Institute for Public Health and Environment, Bilthoven, the Netherlands.
MDEs (effect size: $0.37,95 \%$ CI 0.09 to 0.65 ). This suggests that permanent damage does not routinely occur from a first MDE.

\section{CONCLUSIONS}

Psychological function is mildly impaired for prolonged periods before and after a person's first unipolar MDE. However, permanent damage does not tend to occur from a first MDE.

\section{NOTES}

After an MDE, psychosocial disability may be caused by the state effect (residual depressive symptoms from the episode), the trait effect (continuation of symptoms from before the episode) and/or the scar effect (permanent damage caused by the episode).

\section{Commentary}

t has long been assumed that most cases of depression are episodic and associated with good return to normal premorbid functioning with treatment. Although longitudinal clinical studies have cast doubt on this view, the article by Ormel et al provides partial confirmation, in a general population sample, that significant levels of psychosocial disability not only follow episodes of major depression but antedate them as well. Whether such impairments in functioning are due to a persisting premorbid trait characteristic, subclinical or residual depressive symptoms, an enduring effect of the depressive episode ("scarring"), or indeed some combination of these, is an interesting theoretical question. Of more immediate importance is the need to recognise that depression is more often a chronic condition than is usually supposed.

It is a common clinical experience that, over time, depressive episodes can become more frequent, more severe, and less likely to be triggered by life events. The findings of Ormel et al are consistent with another clinical observation, namely that as depressive episodes recur, the extent of recovery following treatment seems to diminish and the level of residual disability increases.

A question raised by this paper is whether the levels of disability described in their population sample are sufficient to warrant treatment and, if so, how, when, and with what result? The effect sizes found in their study are moderate so that some form of intervention could be justified. Premorbid detection of disability and intervention would not be feasible owing to problems related to defining a threshold for identification and lack of specificity, not to mention cost effectiveness. However, early and assertive evidence-based treatment of depressive episodes may reduce the likelihood of both "scarring" effects and continuation of residual symptoms. Evidence-based treatments for depression are cost effective, but often discontinued prematurely. ${ }^{2}$ It may be that the nature, "dose", and duration of treatment after supposed remission of depressive episodes requires rethinking and greater preparedness of clinicians and patients to continue treatment for a longer time than is currently practiced.

Vaughan J Carr, MD

Centre for Mental Health Studies, University of Newcastle, Newcastle,

Australia

1 Sanderson K, Andrews G, Corry J, et al. Reducing the burden of affective disorders: is evidence-based health care affordable? J Affect Disorders 2003;77:109-25

2 McManus P, Mant A, Mitchell P, et al. Length of therapy with selective serotonin reuptake inhibitors and tricyclic antidepressants in Australia. Aust NZ J Psychiatry 2004;38:450-4. 